\title{
The impact of anorectal malformations on anorectal function and social integration in adulthood: report from a national database
}

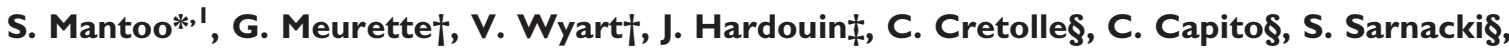 \\ G. Podevin $\uparrow$ and P.-A. Lehurt \\ *Department of Surgery, Khoo Teck Puat Hospital, Singapore City, Singapore, †Digestive and Endocrine Surgery, University Hospital, Nantes, France, \\ $\neq$ Department of Statistics, Faculty of Medicine, University of Nantes, Nantes, France, §Department of Paediatric Surgery, Hopital Necker-Enfants \\ Malades, Université Paris-Descartes, Paris, France and IDepartment of Paediatric Surgery, University Hospital, Angers, France
}

Received 28 August 2012; accepted 15 December 2012; Accepted Article online 7 March 2013

\begin{abstract}
Aim The impact of anorectal malformation (ARM) on bowel function and social, educational and occupational end-points was investigated in adult patients entered on a national database.

Method Data from a national database of adult patients operated on between 1962 and 1999 for ARM were analysed. The database Malformations Ano-rectales et Pelviennes rares (MAREP) was part of a common information system, CEMARA, on rare congenital disorders. A self-administered questionnaire regarding bowel function, academic qualifications, employment and family status was mailed to patients. The type of ARM, subsequent follow-up and management including surgical interventions were retrospectively retrieved from medical records.
\end{abstract}

Results Of 210 adult patients on the registry since 2008, 68 were included in this study. Only three $(8.5 \%)$ had had regular follow-up. All reported some disturbance in bowel function. The fertility rate of 1.5 children per woman did not differ from the general population.

Conclusion Anorectal malformation ARM often leads to suboptimal bowel function in adulthood. This has an impact on social integration.

Keywords Anorectal malformation, sequelae, adults, education, employment, fertility

\section{What is new in this paper?}

The impact of anorectal malformation (ARM) sequelae on anorectal function and social integration in adulthood including educational qualifications and professional achievements has been studied. The MAREP (Malformations Ano-rectales et Pelviennes rares) database allowed us an objective overview of ARM patients at a national level giving a better understanding of the needs of patients affected by this rare but socially important disorder.

\section{Introduction}

Anorectal malformations (ARMs) are part of a wide spectrum of associated malformations in the hindgut area, including bowel, urological, neurological and

Correspondence to: Paul-Antoine Lehur, Clinique de Chirurgie Digestive et Endocrinienne, Institut des Maladies de l'Appareil Digestif (IMAD), University Hospital of Nantes, Hotel Dieu, I Place Alexis Ricordeau, 44093 Nantes, France.

E-mail: paulantoine.lehur@chu-nantes.fr

Some of the results of this study have been presented as an oral poster at the European Society of Colo-Proctology Annual Meeting, 2010, Prague, Czech Republic.

'On HMDP fellowship at University Hospital, Nantes, France. orthopaedic. The functional as well as social outcome of these patients has improved since the 1980s due to a better understanding of pathophysiology and natural history and to standardization of the surgical technique [1]. The introduction of the posterior sagittal approach by Peña and colleagues in the 1980s has been a major development in the management of ARM but longterm follow-up and a dedicated bowel management programme are still essential to achieve an optimal outcome. A bowel management programme starting in adulthood is recommended to improve functional results in terms of continence, so as to offer these patients the best chance of achieving normal physical growth and social integration [2]. The social and functional outcome of children with ARM before the 
introduction of the modern principles of surgery and follow-up has been suboptimal and is largely unreported. Most patients are now adults; they have to cope with the stresses of an adult life and integrate in society.

Recently in France a nationwide programme named Plan National 'Rare Diseases' has been introduced to improve management with the aim of understanding the needs of patients affected by rare but socially important disorders. ARM was eligible for this programme. The aim of this study was to use the national database to evaluate the impact of ARM in adult patients on the functional, social, educational and occupational outcome.

\section{Method}

\section{Study population}

A nationwide database of patients in France born with ARM was established in 2008 having been included in the group of MAREP (Malformations Ano-rectales et Pelviennes rares). It is part of a common information system, CEMARA (Centres Maladies Rares), on rare congenital disorders. CEMARA is dedicated to collecting continuous and complete records of all patients presenting with rare diseases. Data from MAREP have been recorded retrospectively for patients before the establishment of a database through communication with expert centres all over France followed by direct contact with patients, from which thereafter a prospective record is maintained (VW). Our tertiary centre has been approved as the referral centre for adults (18 years old or more) with ARM and for the maintenance of the database by the Commission Nationale de l'Informatique et des Libertés, France. Assessment of the patient's function and its impact on social integration is a major focus of the registry. Subscription is free but entry requires written informed consent from the patient. This study was initiated in line with this objective and all adult patients with at least 1 year of registry follow-up were enrolled.

\section{Patients}

A total of 210 patients had been enrolled in the MAREP database since 2008. Sixty-eight adults were identified and included in the current study. Patient records were retrieved and data were collected on type of ARM, associated other organ or system abnormalities, primary surgical management, function during childhood, and further surgical procedures performed during the follow-up period before or after the registry was set up.
A self-administered questionnaire was mailed to all adult patients, with assessment parameters based on three main end-points as follows.

1 Bowel function including continence (CCF-FI score) and constipation (ROME II criteria) $[3,4]$. Faecal incontinence was defined as mean CCF-FI score of 10 or more (normal 0 ; worst 20) and/or more than one leakage of stools per day. ROME II criteria (two or more of straining; lumpy or hard stools; sensation of incomplete evacuation; sensation of anorectal obstruction/blockage and manual manoeuvres to facilitate $>1 / 4$ of defaecations; and/or $<3$ defaecations per week for at least 12 weeks) had to be satisfied for the definition of constipation.

2 Type of surgical procedure performed in adulthood and associated medical management including pelvic floor retraining, bulk laxatives and rectal enemas. The patients were also asked to report whether they were being specifically followed up for the ARM.

3 Long-term social outcome based on educational qualifications, type and employment status, and marital and family status.

Sixteen patients did not respond or reply to telephone calls or letters. A complete response to all the questionnaires was available for 52 patients who were included in the final analysis. Data for educational qualifications and occupational status were compared with those of the general population, 2010 census data published by the National Institute of Statistics and Economic Studies, France, and adjusted for age and sex to the study patients for statistical analysis.

\section{Statistical analysis}

Quantitative data were expressed as mean with standard deviation. Comparison of the distribution of data between the study patients and the general population was performed using the chi-squared test. Statistical analysis was performed using STATA 12.0 software (StataCorp LP, College Station, Texas, USA) and statistical significance was taken as a $P$-value of $<0.05$.

\section{Results}

The primary procedure for ARM had been carried out between 1962 and 1990 in 39 male and 29 female patients. The mean age was $28( \pm 9)$ years. Based on the Wingspread International Classification, the ARM was high in $21(31 \%)$ and low in $42(62 \%)$ patients [5] (Table 1). It was not possible to assess the specific type of ARM in five (7\%) due to lack of documentation (unknown group). No difference in mean age and the male to female ratio was observed between high or low 
Table I Patient characteristics.

\begin{tabular}{llll}
\hline & \multicolumn{2}{l}{ Type of anorectal malformation* } \\
\cline { 2 - 4 } & High & Low & Unknown \\
\hline Total no. of patients & $21(31)$ & $42(62)$ & $5(7)$ \\
$(n, \%)$ & & & \\
Age (mean \pm SD; years) & $27 \pm 9$ & $29 \pm 8$ & $27 \pm 8$ \\
M:F & $1.6: 1$ & $1.2: 1$ & $1.5: 1$ \\
Multiple malformations & $14(67 \%)$ & $14(33 \%)$ & - \\
$(n, \%)$ & & & \\
No. of patients $($ analysed) & 18 & 32 & 2 \\
Bowel complaints $\dagger(n, \%)$ & $14(78)$ & $23(71)$ & $1(50)$ \\
Incontinence $(n, \%)$ & $10(71)$ & $14(60)$ & - \\
Constipation $(n, \%)$ & $4(29)$ & $9(40)$ & $1(50)$ \\
\hline
\end{tabular}

*Based on Wingspread Classification.

†Bowel complaints in adulthood at time of analysis.

types. Multiple associated malformations [including complete or incomplete VATERL (vertebra-anal-trachea-oesophageal-renal-limb) syndrome] were more common in high $[14(67 \%)]$ than in low $[14(33 \%)]$ ARM.

\section{Bowel function (incontinence and constipation)}

Of 52 patients, only three $(8.5 \%)$ had a dedicated and regular follow-up for bowel management. All patients reported some disturbance in bowel function irrespective of type of ARM and gender. The predominant symptoms were faecal incontinence, obstructed defaecation syndrome symptoms (straining, incomplete evacuation or digitation), rectal or vaginal bulge, and dyspareunia in sexually active patients, in decreasing order of frequency. More than one symptom in any combination was reported in the majority of patients. A few with high or low ARM had only an occasional bowel disturbance and socially acceptable bowel function. Most patients whatever the type of ARM complained of major bowel problems and sought medical treatment in adulthood. Fourteen $(78 \%)$ patients with a high ARM had bowel complaints compared with 23 (71\%) with a low ARM (Table 1). Those with a high ARM had a slightly higher prevalence of continence difficulty than those with a low ARM [10 (71\%) vs $14(60 \%)]$ but chronic constipation was more common in the low compared with the high group [9 (40\%) vs 4 (29\%)].

\section{Surgical interventions in adulthood}

Thirty-one $(46 \%)$ patients had undergone secondary surgical procedures in adulthood with no significant difference between the high $[9(50 \%)]$ and low [19 (59\%)]
Table 2 Secondary and subsequent surgical procedures according to type of ARM.

\begin{tabular}{lll}
\hline Type of ARM $(n)$ & $\begin{array}{l}\text { Secondary } \\
\text { procedures }\end{array}$ & $\begin{array}{l}\text { Subsequent } \\
\text { procedures }\end{array}$ \\
\hline High $(n=9)$ & $\begin{array}{l}\text { MACE 6 } \\
\text { Prolapse repair 2 } \\
\text { SNS 1 }\end{array}$ & $\begin{array}{l}\text { Prolapse repair 1 } \\
\text { MACE 1 }\end{array}$ \\
& MACE 3 & $\begin{array}{l}\text { Prolapse repair 3 } \\
\text { Low }(n=19)\end{array}$ \\
& $\begin{array}{l}\text { Prolapse repair 5 } \\
\text { ABS 6 }\end{array}$ & ABS 2 \\
& Anal graciloplasty 1 & \\
& Stoma 6 & \\
Unknown & ABS 1 & \\
$(n=3)$ & Prolapse repair 2 & \\
\hline
\end{tabular}

ARM, anorectal malformation; MACE, Malone antegrade colonic enema; ABS, artificial bowel sphincter; SNS, sacral nerve stimulation.

ARM types (Table 2). Eight (26\%) with either a high or low ARM had more than one subsequent procedure for failure of a secondary surgical procedure or development of new symptoms. A Malone antegrade colonic enema was performed in 10 patients (eight during adulthood), as either a secondary or subsequent procedure and all were actually using it at the time of the study. Insertion of an artificial bowel sphincter was the most frequent procedure used for faecal incontinence with nine procedures performed. Seven of these had had a good outcome at the time of this assessment and two had persistent soiling or worsening constipation. Rectal prolapse surgery was performed as a secondary procedure in nine patients by perineal $(n=8)$ and abdominal $(n=1)$ approach. Eight of these had surgery for a prolapsed colonic pull-through procedure done in childhood, by a perineal excision with a new colo-anal anastomosis. One patient with a preserved anal sphincter underwent successful sacral nerve stimulation for incontinence. A definitive stoma for refractory symptoms was performed in seven $(23 \%)$ patients as either a secondary or subsequent procedure.

\section{Educational, employment and social outcomes}

All 52 patients analysed including 31 men and 21 women responded to the mailed questionnaire regarding educational, occupational and marital status including the number of children if any. The educational qualifications of ARM patients were compared with the general population at four levels (Fig. 1). A higher enrolment for diploma, graduate and postgraduate courses was observed in the ARM patients resulting in a 


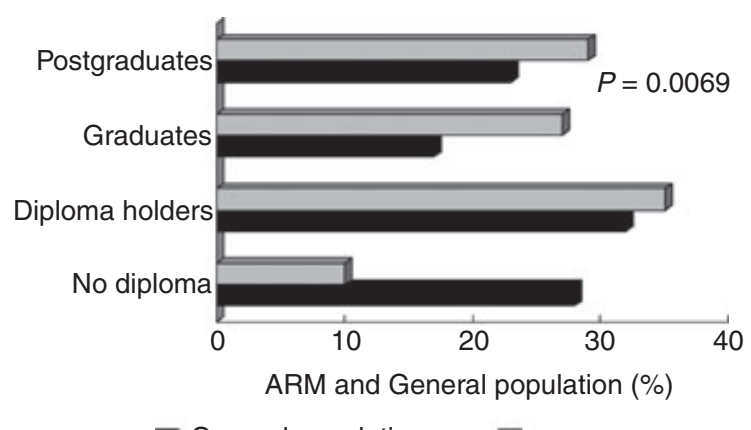

General population

ARM

Figure I Comparison of educational qualifications. ARM, anorectal malformation.

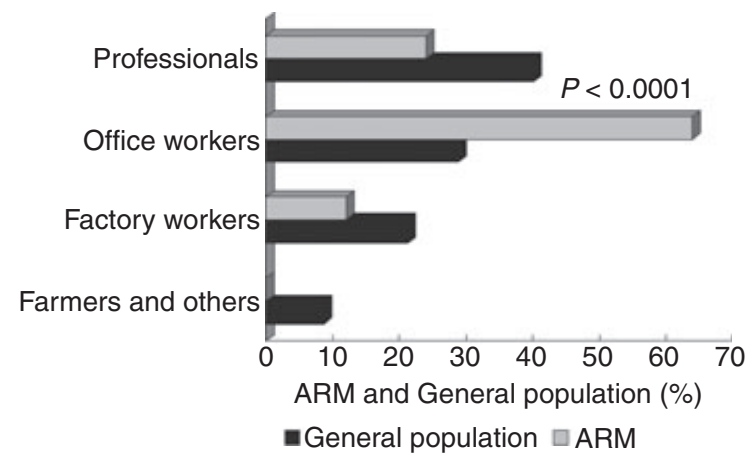

Figure 2 Comparison of occupational status. ARM, anorectal malformation.

higher rate of diploma, graduate and postgraduate qualifications achieved $(P=0.0069)$.

Most $(64 \%)$ of the ARM patients preferred sedentary, low to mid level office jobs compared with $28.9 \%$ of the general population despite having higher educational qualifications $(P<0.0001)$. None of the ARM patients was involved in farming or other manual work and similarly only $12 \%$ were working in factories compared with $21.3 \%$ of the general population (Fig. 2). There was a preference to take less stressful physical and mental jobs by the study cohort even if they were qualified for a higher grade job.

Sexual function, marital status and the number of children of male and female patients are shown in Table 3. Most $(77 \%$ men and $81 \%$ women) were sexually active. Sixty-two per cent of women were married compared with $35 \%$ of men. Five sexually active male patients reported erectile and ejaculatory difficulty and had not married. More $(29 \%)$ women had children than men $(13 \%)$ but none of the female patients with a high ARM had given birth. The fertility rate of 1.5 per female subject did not differ compared with the general population.

\section{Discussion}

Anorectal malformation (ARM) is the most common congenital colorectal defect in the newborn with an incidence of 1 in 3000-5000 live births. Surgery for ARM has been standardized since the 1980s with posterior sagittal anorectoplasty considered as the gold standard technique $[1,6]$. Dedicated bowel management programmes have shown promising results if commenced early $[7,8]$. Functional and social outcomes of children with ARM before the introduction of these modern principles of surgery have been suboptimal and are largely unreported. Secondary surgical procedures for failed or suboptimal primary procedures are unlikely to provide ideal functional results.

Few studies have reported the impact of the longterm consequences of faecal incontinence on quality of life in adults with ARM whereas a number of studies have reported this in children $[9,10]$. A recent study of 62 adults (> 16 years old) and 175 children found that adults reported a significantly lower level of quality of life on subscales of emotional functioning, body image and physical symptoms [11]. The difference was attributed to recent improvements in surgical technique or to the possibility that quality of life in patients with ARM decreases over time. Iwai et al. [12] reported that only one-third of 29 patients (mean age $26 \pm 1$ years) with high- or intermediate-type anomalies after abdominoperineal rectoplasty had some problems with bowel function with faecal soiling as the major complaint responsible for a disturbed social and working life. All the patients in our study reported some bowel dysfunction irrespective of the type of ARM, with faecal incontinence as the most common disturbance affecting social life. We did not find a significant relationship between the severity and type of bowel dysfunction and the type or grade of ARM. However, the assessment for the grade of ARM was incomplete in some of our patients. We speculate that those with a high ARM may have benefited from greater attention to long-term bowel management and psycho-social support.

Due to persistent bowel dysfunction, it is not surprising that multiple surgical procedures were performed in more than half of the patients in the present study. Management of these disorders is complex and debatable $[13,14]$, but it is likely to be better in well equipped centres with experience of functional bowel diseases.

An important observation in the study was that no standardized follow-up was recommended to any patient and all had lost contact with the paediatric team who had operated on them in their childhood. This could be for several reasons including migration, employment and lack of faith in the original surgical 


\begin{tabular}{lccccc}
\hline & $\begin{array}{l}\text { No. of } \\
\text { Type of ARM }\end{array}$ & \multicolumn{2}{l}{ Sexual activity $(n, \%)$} & & Marital \\
& patients & Active & Inactive & & $\begin{array}{l}\text { Children } \\
\text { status }(n, \%)\end{array}$ \\
$(n, \%)$
\end{tabular}

ARM, anorectal malformation.

unit. Only $8.5 \%$ of the patients were under a dedicated long-term follow-up. It is possible that the majority of the patients might have either developed their own coping strategies or simply accepted a poorer quality of life. A dedicated clinic for patients with ARM supervised by a colorectal and a paediatric surgeon with a special interest in ARM has been set up in our centre to better meet the expectations of these patients.

Unique to our study was the focus on the eventual educational and employment status of adults with ARM. This valuable information can be used by physicians to facilitate the counselling of both patients and parents during the course of follow-up. The rate of enrolment for university or higher studies was higher in our study group compared with the general population. Possible explanations include the need to become more independent and self-sufficient earlier in life, resulting in a better ability to prioritize and thus show a greater sense of discipline. However, it was interesting to note that the number of patients who went on to complete longer courses in the university was lower than in the general population. Most of our patients reported that functional disturbances of bowel and bladder with other physical limitations hampered them from taking longer and more intensive courses. This is supported by the observation that most of our study patients were working in sedentary jobs compared with the general population, limiting their subsequent choice of occupation.

The study provides an insight into an important aspect of life for patients with ARM but it has some limitations. The data for all 68 eligible adult patients are not complete with more than $20 \%$ of patients who did not respond to the telephone calls and mails. There can be many reasons. First they might have learned to live with their disability and have no further interest in seeking help. In contrast their failure to respond may
Table 3 Sexual activity, marital status and number of children. have been because their present function was acceptable with no need for further management.

The impact of associated organ-system malformations such as urinary and reproductive systems on the overall outcome has not been presented in detail. It is possible that patients with multiple system abnormalities would have a worse outcome but we were not able to appreciate this difference. Based on the prevalence of ARM, our database certainly does not include all patients in France at present. We are in a constant process of updating our database so that more and more patients will benefit from our dedicated programme and experience.

This study is one of the first efforts to investigate this uncommon but important problem. Its methodology has been kept simple as the data available were basic and failed to include matters of importance such as family social status and mental development. It is hoped that it may pave the way for further reasearch aimed to establish social status.

Anorectal malformation (ARM) is rare with frequent severe sequelae best managed by a multidisciplinary team, including surgeons, paediatricians, psychologists, nutritionists and nurses. An adult patient rather than the parent is able to provide a more reliable picture of the overall outcome, including functional, social, educational and occupational outcomes. Most patients can achieve a high level of educational qualifications and become gainfully employed in adulthood. With adequate support and medical care, social integration is possible.

\section{Sources of funding}

This study was supported and funded by the Ministry of Health, France, under the 'Plan Maladies Rares' (National Programme for Management of Rare Disorders). 


\section{Author contributions}

Surendra Mantoo contributed to study design, analysed the data, wrote the manuscript and contributed to manuscript revisions. Guillaume Meurette worked with the first author during all phases of the study. He contributed to study design and data analysis. Vincent Wyart contributed in communication with patients and collection of data. Jean Hardouin performed statistical analysis. Celia Cretolle contributed to manuscript revisions. Carmen Capito contributed to the conception of the study. Sabine Sarnacki contributed to manuscript revisions. Guillaume Podevin recruited patients and contributed to manuscript revisions. P.-A. Lehur recruited patients and contributed to manuscript revisions.

\section{References}

l Peña A, deVries PA. Posterior sagittal anorectoplasty: important technical considerations and new applications. J Pediatr Surg 1982; 17: 796-811.

2 Peña A, Hong A. Advances in the management of anorectal malformations. Am J Surg 2000; 180: 370-6.

3 Jorge JM, Wexner SD. Etiology and management of fecal incontinence. Dis Colon Rectum 1993; 36: 77-97.

4 Thompson WG, Longstreth GF, Drossman DA, Heaton KW, Irvine EJ, Muller-Lissner SA. Functional bowel disorders and functional abdominal pain. Gut 1999; 45(Suppl 2): 1143-7.

5 Stephens FD, Smith ED. Classification, identification and assessment of surgical treatment of anorectal anomalies. Pediatr Surg Int 1986; 1: 200-5.
6 Peña A (2005). Anorectal Malformations: Operative Pediatric Surgery. Appleton \& Lange, Boston.

7 Grano C, Aminoff D, Lucidi F, Violani C. Long-term disease-specific quality of life in children and adolescent patients with ARM. J Pediatr Surg 2012; 47: 1317-22.

8 Fabbro MA, Chiarenza F, D'Agostino $S$ et al. Anorectal malformations (ARM): quality of life assessed in the functional, urologic and neurologic short and long term followup. Pediatr Med Chir 2011; 33: 182-92.

9 Hartman E, Oort F, Mechteld V et al. Explaining change over time in quality of life of adult patients with anorectal malformations or Hirschsprung's disease. Dis Colon Rectum 2005; 49: 96-103.

10 Nixon HH, Puri P. The results of treatment of anorectal anomalies: a thirteen to twenty year follow-up. J Pediatr Surg 1977; 12: 27-37.

11 Grano C, Aminoff D, Lucidi F, Violani C. Disease-specific quality of life in children and adults with anorectal malformations. Pediatr Surg Int 2010; 26: 151-5.

12 Iwai N, Deguchi E, Kimura O, Kubota Y, Ono S, Shimadera S. Social quality of life for adult patients with anorectal malformations. J Pediatr Surg 2007; 42: 313-7.

13 Wong MT, Meurette G, Wyart V, Glemain P, Lehur PA. The artificial bowel sphincter: a single institution experience over a decade. Ann Surg 2011; 254: 951-6.

14 Wexner SD, Jin HY, Weiss EG, Nogueras JJ, Li VK. Factors associated with failure of the artificial bowel sphincter: a study of over 50 cases from Cleveland Clinic Florida. Dis Colon Rectum 2009; 52: 1550-7. 